\title{
Biologia alimentar de quatro espécies simpátricas de Cheirodontinae (Characiformes, Characidae) do rio Ceará Mirim, Rio Grande do Norte
}

\author{
Tatiana S. Dias \& Clarice B. Fialho
}

Laboratório de Ictiologia, Departamento de Zoologia, Programa de Pós-Graduação em Biologia Animal, Universidade Federal do Rio Grande do Sul, Av. Bento Gonçalves, 9500, Prédio 43435, 91501-970 Porto Alegre, RS, Brasil. (ts_dias@yahoo.com.br)

\begin{abstract}
Feeding biology of four sympatric Cheirodontinae species (Characiformes, Characidae) from Ceará Mirim River, Rio Grande do Norte, Brazil. This study was carried in Ceará Mirim River, State of Rio Grande do Norte, Brazil. At this location, the river shows high water transparency and predominance of aquatic macrophytes in the margins. This study aims to describe the feeding habits and verify the presence of feeding overlap in the diet of four sympatric species of Cheirodontinae: Compsura heterura Eigenmann, $1915(\mathrm{n}=452)$, Serrapinnus heterodon (Eigenmann, 1915) $(\mathrm{n}=473)$, S. piaba (Lütken, 1875) $(\mathrm{n}=509)$ and Serrapinnus sp. A $(n=313)$. The specimens were collected monthly, from April 2001 to April 2002, using seine nets. The stomach contents were analyzed using the frequency of occurrence, percentage composition and an alimentary importance index. The feeding overlap was calculated through the index of Morisita between pairs of species. The species did not showed seasonal variation in the diet. Algae, vegetal matter, microcrustaceans and autochthonous insects were predominant in the species diet. Serrapinnus heterodon and Serrapinnus sp. A presented omnivorous feeding behaviour, while $C$. heterura and $S$. piaba showed omnivorous feeding behaviour with tendency to herbivory. In addition, the species showed high feeding overlap due to the consumption of similar items. These results suggest that food resources are abundant and enough to be partioned by these four omnivorous sympatric species.
\end{abstract}

KEYWORDS. Northeast Brazil, omnivory, feeding overlap, fishes.

RESUMO. Este estudo foi realizado no rio Ceará Mirim, Estado do Rio Grande do Norte. No local de coleta, este rio apresenta águas transparentes e predominância de macrófitas aquáticas nas margens. Este estudo teve como objetivos descrever os hábitos alimentares e verificar a existência de sobreposição alimentar na dieta de quatro espécies simpátricas de Cheirodontinae: Compsura heterura Eigenmann, $1915(\mathrm{n}=452)$, Serrapinnus heterodon (Eigenmann, 1915) $(\mathrm{n}=473)$, S. piaba (Lütken, 1875) $(\mathrm{n}=509)$ e Serrapinnus $\mathrm{sp}$. A $(\mathrm{n}=313)$. Os espécimes foram coletados mensalmente, entre abril de 2001 e abril de 2002 , com redes de arrasto. Os conteúdos estomacais foram analisados através dos métodos de frequência de ocorrência, composição percentual e índice de importância alimentar. A sobreposição alimentar foi calculada entre os pares de espécies através do índice de Morisita. As espécies não mostraram variação sazonal na dieta. Algas, matéria vegetal, microcrustáceos e insetos autóctones foram predominantes na dieta das espécies. Serrapinnus heterodon e Serrapinnus sp. A apresentaram hábito alimentar onívoro, enquanto C. heterura e S. piaba apresentaram o hábito alimentar onívoro com tendência à herbivoria. Além disso, altos valores de sobreposição alimentar entre as espécies foram observados em decorrência do consumo de itens similares. Estes resultados sugerem que os recursos alimentares são abundantes e suficientes para serem partilhados por estas quatro espécies onívoras em simpatria.

PALAVRAS-CHAVE. Nordeste brasileiro, onivoria, sobreposição alimentar, peixes.

Estudos sobre a utilização do alimento, além de ser um campo importante para a discussão de aspectos teóricos, como a substituição das espécies através dos componentes espacial, temporal e trófico do nicho (SCHÖENER, 1974), permitem o conhecimento da biologia, da interação entre as espécies e da organização do ecossistema (HERRÁN, 1988).

A família Characidae é a maior e mais complexa entre os peixes neotropicais (BRITSKI et al., 1999; NELSON, 2006), apresentando diversas formas corporais, o que lhes permite ocupar diferentes habitats e desenvolver estratégias alimentares variadas (LowE-McConNeLL, 1999; GraÇA \& PaVAnelli, 2007). Entre as subfamílias de Characidae, Cheirodontinae é uma das mais conhecidas em termos de taxonomia e filogenia, contando com cerca de 50 espécies válidas (Malabarba, 2003). São geralmente abundantes em águas lênticas e em regiões de baixas latitudes, ocorrendo na maioria das drenagens das Américas Central e do Sul (Malabarba, 1998). Dentro desta família encontram-se Compsura heterura Eigenmann, 1915, Serrapinnus heterodon (Eigenmann,
1915), S. piaba (Lütken, 1875) e Serrapinnus sp. A, espécies que vivem em simpatria no rio Ceará Mirim, Rio Grande do Norte.

Alguns estudos realizados sobre os hábitos alimentares de espécies de Cheirodontinae demonstram sua preferência alimentar por itens de origem autóctone (Destefanis \& Freyre, 1972; ANGermeier \& Karr, 1983; Escalante, 1987; Jacobo \& Veron, 1995; Alvim et al., 1997; LuIz et al., 1998; GASPAR DA LuZ et al., 2001; BISTONI \& Hued, 2002; CasatTi et al., 2003; CAzorla et al., 2003; Petry et al., 2003; Hirano \& AzeVEdo, 2007). No entanto, apesar da sua ampla distribuição (MALABARBA, 2003), nenhum trabalho foi publicado até o momento sobre a biologia alimentar de espécies de Cheirodontinae do nordeste do Brasil, o que evidencia a necessidade de conhecimento mais aprofundado sobre os hábitos destes pequenos caracídeos.

Devido ao fato de a maioria dos peixes possuir larga tolerância a diferentes tipos de habitats e certa plasticidade alimentar, estes podem partilhar muitos recursos do seu ambiente com outras espécies (LowE- 
McConnell, 1999). Segundo Abrams (1980), a sobreposição de nicho ocorre quando duas ou mais espécies utilizam um mesmo recurso do ambiente. Segundo o princípio da exclusão competitiva, quando espécies filogeneticamente aparentadas e com necessidades ecológicas semelhantes coexistem em um ambiente, estas podem competir entre si e resultar na exclusão da espécie menos adaptada (GAUSE, 1934; Hardin, 1960). Porém, se indivíduos que exploram os mesmos recursos em uma comunidade coexistem, então podemos supor que eles exploram estes recursos de forma diferenciada, ou que a competição pode não se manifestar se os recursos forem abundantes no ambiente (Margalef, 1986; Pianka, 1994).

Considerando que as semelhanças morfológicas tendem a aumentar a competição interespecífica, esperase que as espécies de Cheirodontinae coexistentes e com morfologia similar explorem diferentemente o recurso alimentar disponível no ambiente estudado. Assim, este trabalho tem como objetivo descrever a dieta de quatro espécies simpátricas de Cheirodontinae do rio Ceará Mirim, estabelecendo os seus hábitos alimentares e verificando a presença de sobreposição alimentar entre suas dietas, a fim de verificar se a coexistência está associada à partilha de recursos alimentares entre elas.

\section{MATERIAL E MÉTODOS}

As coletas foram realizadas no rio Ceará Mirim (5'37'47' 'S, 35³7'9' W), situado no município de Taipu, distrito de Umari, Rio Grande do Norte. Este rio, com aproximadamente $2.770 \mathrm{~km}^{2}$ de extensão, forma a principal bacia hidrográfica do Estado. No local de coleta o rio apresenta fundo arenoso e profundidade máxima de 1,20 m. Sua água é transparente, alternando áreas de correnteza média e áreas de remanso. Apresenta vegetação flutuante e submersa em abundância, sendo as margens contornadas por espécies herbáceas. As quatro espécies aqui estudadas foram coletadas junto às margens do rio Ceará Mirim (Luiz R. Malabarba, com. pess.), onde se encontra uma grande quantidade de macrófitas.

As coletas foram realizadas mensalmente, entre abril de 2001 e abril de 2002. Os espécimes foram capturados com redes de arrasto com malha de $5 \mathrm{~mm}$ entre nós adjacentes. Os indivíduos foram fixados, ainda em campo, em formalina $10 \%$. Em laboratório, os peixes foram transferidos para álcool $70^{\circ} \mathrm{GL}$ e, de cada indivíduo, foram registrados os dados de comprimento padrão $(\mathrm{CP})$ em milímetros, peso total e peso do estômago em gramas. Espécimes-testemunho foram depositados na coleção ictiológica do Departamento de Zoologia da Universidade Federal do Rio Grande do Sul (C. heterura lotes UFRGS 8978, 8979, 9213; S. heterodon lote UFRGS 8793; S. piaba lotes UFRGS 5807, 8794; Serrapinnus sp. A lotes UFRGS 9216-9227).

Os conteúdos estomacais foram analisados através de microscópio estereoscópico e, quando necessário, de microscópio óptico. A identificação dos itens alimentares foi realizada até o menor nível taxonômico possível com o auxílio de bibliografia específica (NEDHAM \& NeDHAM, 1978; Lopretto \& Tell, 1995). Os resultados foram agrupados por estações do ano: outono (março, abril e maio), inverno (junho, julho e agosto), primavera (setembro, outubro e novembro) e verão (dezembro, janeiro e fevereiro).

A dieta foi definida pelos métodos de frequência de ocorrência e composição percentual, segundo HyNES (1950). O percentual de frequência de ocorrência foi calculado através da ocorrência de cada categoria alimentar no conjunto de estômagos com alimento. Já a composição percentual considera a proporção de quadrículas ocupadas por cada item alimentar em um papel milimetrado em relação ao número total de quadrículas ocupadas por todos os itens.

Os itens alimentares foram agrupados em categorias taxonômicas ou ecológicas amplas, sendo que os insetos foram classificados como autóctones quando originários do ambiente aquático (larvas, pupas), ou alóctones quando originários do ambiente terrestre. As categorias consideradas foram: microcrustáceos, Collembola, insetos autóctones, insetos alóctones, Arachnida, escamas de peixes, algas, matéria vegetal e matéria orgânica.

A importância de cada uma destas categorias alimentares na dieta das espécies foi calculada através de uma escala semi-quantitativa de abundância, onde a contribuição de cada categoria é estimada de acordo com a área que a mesma ocupa em relação ao conteúdo total. Foi utilizada a seguinte escala proposta por GRANADOLoRENCIO \& GARCIA-Novo (1986): 0 - ausente; 1 - escasso (até $25 \%$ ); 2 - frequente (acima de $25 \%$ a $50 \%$ ); 3 muito frequente (acima de $50 \%$ a $75 \%$ ); 4 - abundante (acima de $75 \%$ ). Segundo esta escala, calculou-se o índice de importância alimentar (IIA) utilizando-se a seguinte fórmula (Granado-LoRENCIO \& Garcia-Novo, 1986): $\mathrm{IIA}=\sum\left[\left(\mathrm{X}_{\mathrm{k}} \cdot \mathrm{K}\right) /(\mathrm{n}-1)\right]$, onde $\mathrm{Xk}$ corresponde à frequência de ocorrência de um determinado item da dieta $X_{\mathrm{i}}$ com categoria K; K é a categoria de abundância $(0,1,2,3$ ou 4), e n corresponde ao número de categorias da escala. De acordo com Guillen \& GRANADO (1984), o valor deste índice para cada item alimentar varia de 0 a 1 , sendo considerado alimento principal se o valor for superior ou igual a 0,3 ; alimento adicional se o valor estiver entre 0,3 e 0,15 , inclusive; e alimento acidental se o valor for menor que 0,15 .

O grau de sobreposição alimentar foi calculado através do índice simplificado de Morisita (KREBS, 1989), utilizando-se os valores do índice de importância alimentar de cada item. Os valores do índice de sobreposição alimentar calculados variam de zero quando as dietas são completamente distintas, a um, quando apresentam a mesma composição de importância de seus itens. Segundo ZARET \& RAND (1971), um valor igual ou maior que 0,6 indica uma sobreposição significativa nas dietas.

As dietas das espécies foram agrupadas empregando-se uma análise de Cluster usando a distância euclidiana pelo método de similaridade através do programa XLStat. A sazonalidade na ingestão de 
determinadas categorias foi testada através de uma análise de variância pelo teste Kruskal-Wallis (ZAR, 1999). Ambos os testes foram realizados utilizando-se como fator as estações do ano e, como variável, os valores do índice de importância alimentar.

\section{RESULTADOS}

Neste estudo foram analisados os conteúdos estomacais de 452 espécimes de $C$. heterura (CP 17,9336,90 mm), 473 de S. heterodon (CP 13,34-33,42 mm), 509 de $S$. piaba (CP 20,22-30,18 mm) e 313 de Serrapinnus sp. $\mathrm{A}(\mathrm{CP} 18,42-33,61 \mathrm{~mm})$.

Os resultados indicaram, para $C$. heterura, uma dieta composta em grande parte por matéria vegetal e algas, especialmente Chlorophyta (Tab. I). Os itens de origem vegetal obtiveram os valores mais altos do índice de importância alimentar (Tab. II) e representaram cerca de $80 \%$ da composição percentual da dieta desta espécie (Fig. 1).

Para S. heterodon, altas frequências de insetos autóctones, algas, matéria vegetal e microcrustáceos foram observadas (Tab. I). O índice de importância alimentar (Tab. II) mostrou valores elevados para insetos autóctones, algas, microcrustáceos e matéria vegetal, sendo os dois primeiros itens considerados alimentos principais e os dois últimos alimentos adicionais, em quase todo o período estudado. Através da composição percentual (Fig. 1), verificou-se que esta espécie alimentou-se em maior proporção de itens de origem vegetal $(65 \%)$, enquanto os itens de origem animal somam pouco mais de $30 \%$.
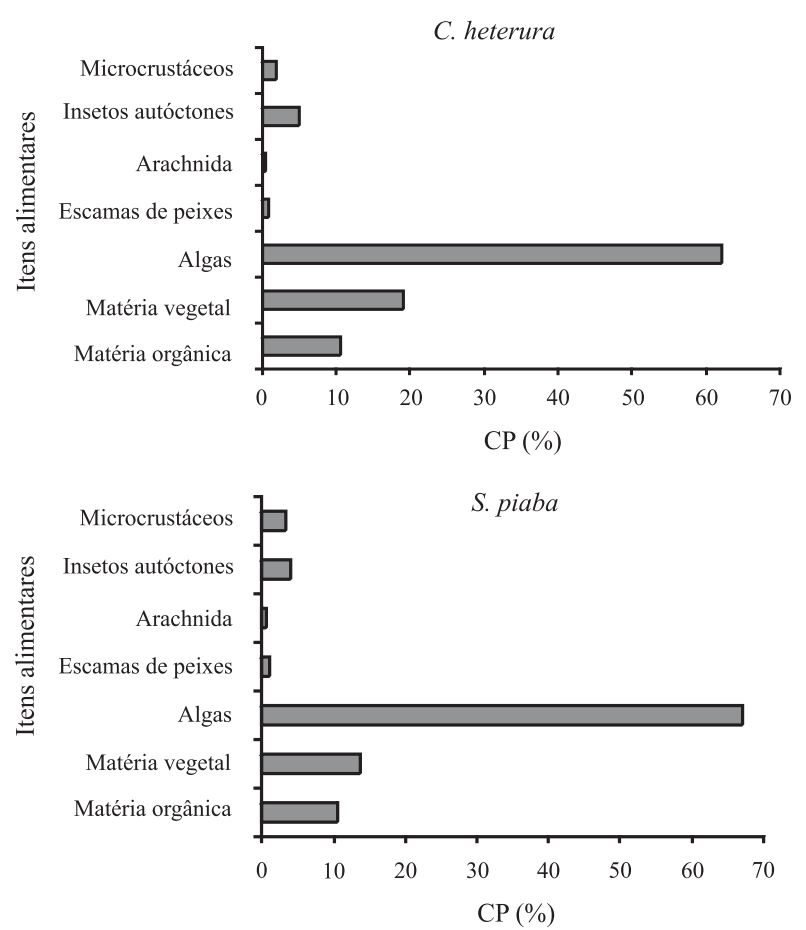

Serrapinnus piaba apresentou altas frequência e abundância de algas e matéria vegetal na sua dieta (Tab. I; Fig. 1). A análise do índice de importância alimentar (Tab. II) indicou que as algas constituíram o principal alimento na dieta desta espécie.

A dieta de Serrapinnus sp. A teve elevada contribuição de itens de origem vegetal (cerca de 67 \%) enquanto os itens de origem animal contribuem com aproximadamente $27 \%$ (Tab. I; Fig. 1). Através do índice de importância alimentar (Tab. II) observou-se os itens de origem vegetal foram alimentos principais ou adicionais em quase todo o período estudado. Itens como insetos autóctones e microcrustáceos também demonstraram sua importância, sendo o primeiro principal e o segundo adicional em algumas estações.

O índice de sobreposição alimentar entre as espécies (Tab. III) apresentou valores elevados durante todo o período analisado, indicando que as espécies utilizam recursos alimentares semelhantes.

Da mesma forma, a análise de Cluster (Fig. 2) mostrou que as espécies são altamente similares com relação as suas dietas, sendo divididas em duas categorias tróficas: onívoras ( $S$. heterodon e Serrapinnus sp. A) e onívoras com tendência a herbivoria ( $C$. heterura e S. piaba).

Através da análise de variância pelo teste Kruskal-Wallis verificou-se a ausência de sazonalidade na dieta de $C$. heterura $(\mathrm{H}=0,2016$; $\mathrm{p}=0,9952)$, S. heterodon $(\mathrm{H}=0,2599 ; \mathrm{p}=0,9923), S$. piaba $(\mathrm{H}=0,8264 ; \mathrm{p}=0,93498)$ e Serrapinnus sp. A $(\mathrm{H}=0,1393 ; \mathrm{p}=0,9977)$.
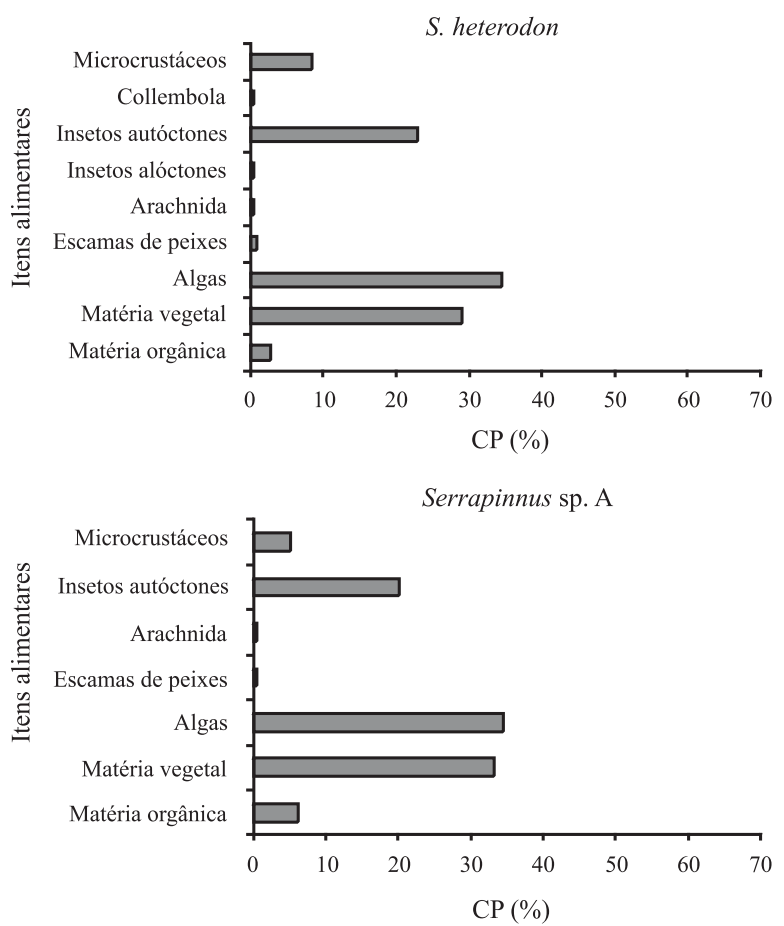

Figura 1. Composição percentual (CP) dos itens alimentares ingeridos por Compsura heterura Eigenmann, 1915 ( $\mathrm{n}=452)$, Serrapinnus heterodon (Eigenmann, 1915) $(\mathrm{n}=473)$, Serrapinnus piaba (Lütken, 1875) $(\mathrm{n}=509)$ e Serrapinnus $\mathrm{sp} . \mathrm{A}(\mathrm{n}=313)$, coletados no rio Ceará Mirim, Rio Grande do Norte, durante o período de abril de 2001 a abril de 2002. 
Tabela I. Frequência de ocorrência dos itens alimentares ingeridos por Compsura heterura Eigenmann, 1915 (n= 452), Serrapinnus heterodon (Eigenmann, 1915) $(\mathrm{n}=473)$, Serrapinnus piaba (Lütken, 1875) $(\mathrm{n}=509)$ e Serrapinnus sp. A $(\mathrm{n}=313)$, coletados no rio Ceará Mirim, Rio Grande do Norte, durante o período de abril de 2001 a abril de 2002. (I, inverno; O, outono; P, primavera; V, verão).

\begin{tabular}{|c|c|c|c|c|c|c|c|c|c|c|c|c|c|c|c|c|c|c|c|c|}
\hline \multirow[b]{2}{*}{ Itens alimentares } & \multicolumn{5}{|c|}{ C. heterura } & \multicolumn{5}{|c|}{ S. heterodon } & \multicolumn{5}{|c|}{ S. piaba } & \multicolumn{5}{|c|}{ Serrapinnus sp. A } \\
\hline & $\mathrm{O}$ & $\mathrm{I}$ & $\mathrm{P}$ & $\mathrm{V}$ & $\mathrm{O}$ & $\mathrm{O}$ & $\mathrm{I}$ & $\mathrm{P}$ & $\mathrm{V}$ & $\mathrm{O}$ & $\mathrm{O}$ & I & $\mathrm{P}$ & $\mathrm{V}$ & $\mathrm{O}$ & $\mathrm{O}$ & I & $\mathrm{P}$ & $\mathrm{V}$ & $\mathrm{O}$ \\
\hline Crustacea & 9,3 & 15,4 & 15,4 & 9,3 & 2,6 & 82,9 & 91,2 & 85 & 93,2 & 67,9 & 57 & 72,9 & 79,7 & 69,8 & 35 & 64,4 & 50 & 65,8 & 36,4 & 20,2 \\
\hline Collembola & & & & & & 2,4 & & & & & & & & & & & & & & \\
\hline Insetos autóctones & 41,3 & 52,3 & 25,6 & 17 & 11,7 & 90,2 & 98,3 & 83 & 71,8 & 66,7 & 32,9 & 55,1 & 46 & 26,9 & 15 & 40,7 & 85,3 & 72,8 & 59,1 & 20,2 \\
\hline Diptera & 8 & 9,2 & 0,9 & 5,1 & 5,2 & 29,3 & 18,4 & 16,7 & 26,5 & 16 & 12,7 & 16,1 & 14,2 & 16,8 & 5 & 5,1 & 20,6 & 16,7 & & 6 \\
\hline Ephemeroptera & 12 & 10,7 & 7,7 & 2,5 & 2,6 & 34,1 & 21,1 & 12,5 & 11,1 & 13,6 & 6,3 & 11,9 & 5,3 & 2,5 & & 6,8 & 41,2 & 21,1 & 27,3 & \\
\hline Lepidoptera & 1,3 & & & & & & & 0,8 & & & & & & 0,8 & 1,3 & & & & & \\
\hline Trichoptera & 2,7 & 1,5 & 0,9 & & 1,3 & & 3,5 & 6,7 & 6 & 4,9 & 1,3 & 0,9 & 0,9 & & & 1,7 & 8,8 & 14,9 & 4,5 & 1,2 \\
\hline Partes de insetos & 29,3 & 47,7 & 18 & 13,6 & 6,5 & 82,9 & 94,7 & 76,7 & 70,1 & 50,6 & 20,3 & 46,6 & 32,7 & 7,6 & 8,8 & 37,3 & 82,4 & 68,4 & 59,1 & 16,7 \\
\hline Insetos alóctones & & & & & & & 0,9 & & & 1,2 & & & & & & & & & & \\
\hline Odonata & & & & & & & 0,9 & & & 1,2 & & & & & & & & & & \\
\hline Arachnida & & & & 0,9 & & & 1,8 & 2,5 & 2,6 & 1,2 & 3,8 & 0,9 & 1,8 & & 2,5 & & & 5,3 & 13,6 & 1,2 \\
\hline Acari & & & & 0,9 & & & 1,8 & 2,5 & 2,6 & 1,2 & 3,8 & 0,9 & 1,8 & & 2,5 & & & 5,3 & 13,6 & 1,2 \\
\hline Escamas de peixes & 5,3 & 4,6 & 2,6 & 4,2 & 2,6 & 14,6 & 12,3 & 4,2 & 6,8 & 11,1 & 11,4 & 14,4 & 8 & 13,5 & 2,5 & 5,1 & 5,9 & 2,6 & 9,1 & 4,8 \\
\hline Algas & 98,7 & 86,2 & 80,3 & 95,8 & 96,1 & 68,3 & 44,7 & 62,5 & 88 & 93,8 & 89,9 & 94,9 & 73,5 & 98,3 & 98,8 & 91,5 & 50 & 38,6 & 59,1 & 85,7 \\
\hline Bacillariophyta & 1,3 & 15,4 & 6,8 & 1,7 & 3,9 & & 3,5 & 2,5 & 1,7 & & 3,8 & 8,5 & 3,5 & & & 1,7 & 8,8 & 14 & 4,5 & 3,6 \\
\hline Chlorophyta & 92 & 75,4 & 72,7 & 89,8 & 88,3 & 56,1 & 42,1 & 56,7 & 82,9 & 92,6 & 88,6 & 90,7 & 67,3 & 97,5 & 95 & 86,4 & 44,1 & 29 & 50 & 78,6 \\
\hline Chrysophyta & 32 & 7,7 & 14,5 & 2,5 & 13 & & & 3,3 & 8,5 & 24,7 & 15,2 & 11 & 11,5 & 4,2 & 3,8 & 3,4 & 2,9 & 5,3 & 4,5 & 9,5 \\
\hline Cyanophyta & 1,3 & & & 7,6 & 24,7 & & 0,9 & 1,7 & 0,9 & 4,9 & & 2,5 & 4,4 & 4,2 & 31,3 & & & 3,5 & 4,5 & 10,7 \\
\hline Rhodophyta & 25,3 & 23,1 & 43,6 & 44,9 & 23,4 & 29,3 & 7 & 18,3 & 42,7 & 24,7 & 35,4 & 25,4 & 30,1 & 28,6 & 6,3 & 30,5 & 5,9 & 15,8 & 27,3 & 14,3 \\
\hline Matéria vegetal & 58,7 & 63,1 & 87,2 & 86,4 & 80,5 & 90,2 & 68,4 & 92,5 & 82,1 & 77,8 & 78,5 & 69,5 & 89,4 & 76,5 & 92,5 & 86,4 & 61,8 & 86 & 59,1 & 89,3 \\
\hline Matéria orgânica & 37,3 & 52,3 & 58,1 & 42,4 & 53,3 & 36,6 & 9,6 & 3,3 & 28,2 & 21 & 74,7 & 78,8 & 80,5 & 53,8 & 26,3 & 66,1 & 14,7 & 15,8 & 13,6 & 33,3 \\
\hline $\mathrm{n}$ & 75 & 65 & 117 & 118 & 77 & 41 & 114 & 120 & 117 & 81 & 79 & 118 & 113 & 119 & 80 & 59 & 34 & 114 & 22 & 84 \\
\hline
\end{tabular}

Tabela II. Índice de importância alimentar dos itens alimentares ingeridos por Compsura heterura Eigenmann, 1915 (n= 452), Serrapinnus heterodon (Eigenmann, 1915) $(\mathrm{n}=473)$, Serrapinnus piaba (Lütken, 1875) $(\mathrm{n}=509)$ e Serrapinnus $\mathrm{sp}$. A $(\mathrm{n}=313)$, coletados no rio Ceará Mirim, Rio Grande do Norte, durante o período de abril de 2001 a abril de 2002. Alimento principal= valor superior ou igual a 0,3; alimento adicional $=$ valor entre 0,3 e 0,15 , inclusive; alimento acidental= valor menor que 0,15 (I, inverno; O, outono; P, primavera; $\mathrm{V}$, verão).

\begin{tabular}{|c|c|c|c|c|c|c|c|c|c|c|c|c|c|c|c|c|c|c|c|c|}
\hline \multirow[b]{2}{*}{ Itens alimentares } & \multicolumn{5}{|c|}{ C. heterura } & \multicolumn{5}{|c|}{ S. heterodon } & \multicolumn{5}{|c|}{ S. piaba } & \multicolumn{5}{|c|}{ Serrapinnus sp. A } \\
\hline & $\mathrm{O}$ & $\mathrm{I}$ & $\mathrm{P}$ & $\mathrm{V}$ & $\mathrm{O}$ & $\mathrm{O}$ & I & $\mathrm{P}$ & $\mathrm{V}$ & $\mathrm{O}$ & $\mathrm{O}$ & I & $\mathrm{P}$ & $\mathrm{V}$ & $\mathrm{O}$ & $\mathrm{O}$ & I & $\mathrm{P}$ & $\mathrm{V}$ & $\mathrm{O}$ \\
\hline Microcrustáceos & 0,02 & 0,04 & 0,04 & 0,02 & 0,01 & 0,22 & 0,27 & 0,26 & 0,28 & 0,17 & 0,14 & 0,19 & 0,21 & 0,18 & 0,09 & 0,17 & 0,19 & 0,23 & 0,09 & 0,06 \\
\hline Collembola & & & & & & 0,01 & & & & & & & & & & & & & & \\
\hline Insetos autóctones & 0,14 & 0,18 & 0,07 & 0,05 & 0,03 & 0,44 & 0,66 & 0,31 & 0,32 & 0,23 & 0,09 & 0,17 & 0,12 & 0,07 & 0,04 & 0,13 & 0,56 & 0,36 & 0,38 & 0,06 \\
\hline Insetos alóctones & & & & & & & 0,01 & & & 0,01 & & & & & & & & & & \\
\hline Arachnida & & & & 0,01 & & & 0,01 & 0,01 & 0,01 & 0,01 & 0,01 & 0,01 & 0,01 & & 0,01 & & & 0,01 & 0,03 & 0,01 \\
\hline Escamas de peixes & 0,02 & 0,01 & 0,01 & 0,01 & 0,01 & 0,05 & 0,03 & 0,01 & 0,02 & 0,03 & 0,03 & 0,04 & 0,02 & 0,04 & 0,02 & 0,01 & 0,02 & 0,02 & 0,02 & 0,01 \\
\hline Algas & 0,77 & 0,61 & 0,49 & 0,73 & 0,75 & 0,27 & 0,21 & 0,32 & 0,55 & 0,74 & 0,63 & 0,71 & 0,45 & 0,87 & 0,82 & 0,55 & 0,25 & 0,21 & 0,43 & 0,51 \\
\hline Matéria ve & 0,16 & 0,27 & 0,48 & 0,28 & 0,29 & 0,43 & 0,23 & 0,53 & 0,26 & 0,24 & 0,21 & 0,23 & 0,46 & 0,21 & 0,37 & 0,28 & 0,29 & 0,54 & 0,29 & 0,55 \\
\hline Matéria orgânica & 0,19 & 0,25 & 0,25 & 0,21 & 0,19 & 0,11 & 0,03 & 0,11 & 0,08 & 0,08 & 0,45 & 0,28 & 0,33 & 0,18 & 0,07 & 0,35 & 0,05 & 0,06 & 0,03 & 0,14 \\
\hline
\end{tabular}

Tabela III. Índice de sobreposição alimentar entre os pares de espécies coletadas no rio Ceará Mirim, Rio Grande do Norte, durante o período de abril de 2001 a abril de 2002 (I, inverno; O, outono; $\mathrm{P}$, primavera; V, verão).

\begin{tabular}{lccccc}
\hline Pares de espécies & $\mathrm{O}$ & $\mathrm{I}$ & $\mathrm{P}$ & $\mathrm{V}$ & $\mathrm{O}$ \\
\hline C. heterura - S. heterodon & 0,61 & 0,61 & 0,86 & 0,85 & 0,95 \\
C. heterura - S. piaba & 0,92 & 0,97 & 0,96 & 0,97 & 0,98 \\
C. heterura - Serrapinnus sp. A & 0,91 & 0,69 & 0,81 & 0,81 & 0,91 \\
S. heterodon - S. piaba & 0,65 & 0,61 & 0,89 & 0,86 & 0,97 \\
S. heterodon - Serrapinnus sp. A & 0,77 & 0,92 & 0,98 & 0,91 & 0,85 \\
S. piaba - Serrapinnus sp. A & 0,98 & 0,67 & 0,84 & 0,74 & 0,91 \\
\hline
\end{tabular}

\section{DISCUSSÃO}

A ocorrência de uma espécie em um determinado local depende, entre outros fatores, da disponibilidade de recursos alimentares e, consequentemente, os sítios de alimentação coincidem com os locais de maior densidade de espécies (HARTz et al., 2000). O local de coleta das espécies aqui estudadas, junto às margens do rio Ceará Mirim, onde se encontra uma grande quantidade de macrófitas, é, possivelmente, o local preferencial de alimentação destes Cheirodontinae. A presença de Cheirodontinae associados a macrófitas e alimentandose nestes locais foram reportados em vários trabalhos 


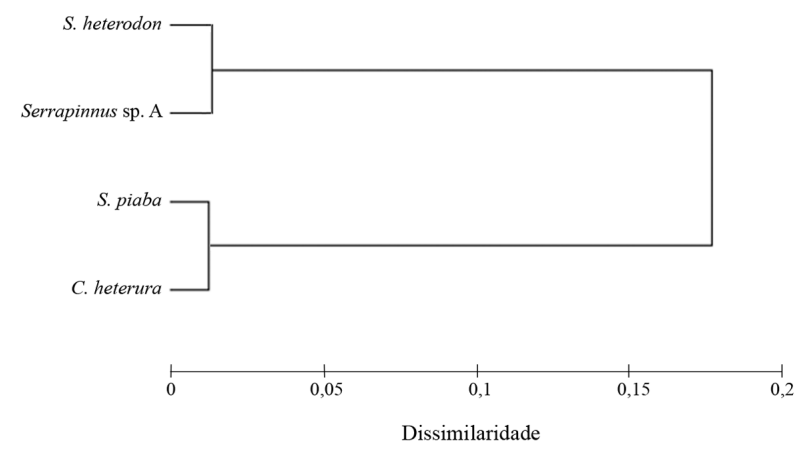

Figura 2. Dendrograma de dissimilaridade entre as espécies de Cheirodontinae, montado a partir dos valores do índice de importância alimentar dos itens consumidos, utilizando a distância euclidiana e o método de aglomeração de Ward.

(Delariva et al., 1994; Casatti et al., 2003; Petry et al., 2003; SмiтH et al., 2003; CASATTI, 2004), onde os autores comentam que a preferência das espécies de peixes por estes habitats está na grande quantidade de algas e invertebrados aquáticos encontrados nas partes submersas desta vegetação. Além disso, todos os métodos de análise empregados neste estudo demonstraram a importância dos itens de origem autóctone na alimentação destas espécies de Cheirodontinae, especialmente algas, matéria vegetal, microcrustáceos e insetos autóctones.

As espécies de Cheirodontinae aqui estudadas apresentaram um reduzido espectro alimentar, sendo essa característica também observada na dieta de Cheirodon interruptus (Jenyns, 1842) (CAZORLA et al., 2003) no rio Sauce Grande (Argentina), S. notomelas (Eigenmann, 1915) no reservatório Rosana (CASATTI et al., 2003; Pelicice \& Agostinho, 2006) e Heterocheirodon yatai (Casciotta, Miquelarena \& Protogino, 1992) (HIRANO \& AzEvedo, 2007) em tributários do rio Ibicui. Acredita-se que este reduzido espectro alimentar possa ocorrer pela elevada disponibilidade destes itens no ambiente (WeATHERLEY, 1972) ou ser simplesmente reflexo de uma preferência desta subfamília pela obtenção de um número restrito de itens em sua dieta.

As espécies aqui estudadas foram primeiramente classificadas na categoria alimentar onívora de acordo com os trabalhos de SABINO \& CASTRO (1990), GASPAR DA Luz et al. (2001) e Luz-Agostinho et al. (2006), onde o termo onívoro é usado para caracterizar a dieta de indivíduos que utilizam alimento animal e vegetal em partes relativamente equilibradas. No entanto, ao observar o predomínio dos itens de origem vegetal nas dietas de $C$. heterura e S. piaba, optou-se por classificar estas espécies como onívoras com tendência a herbivoria, enquanto S. heterodon e Serrapinus sp. A foram consideradas estritamente onívoras. Estudos realizados com outras espécies de Cheirodontinae mostraram resultados similares relatando o hábito alimentar onívoro para S. notomelas (ARCIFA et al., 1988) na represa Americana, S. heterodon (Esteves, 2000) em uma lagoa marginal do rio Mogi-Guaçu, Odontostilbe microcephala Eigenmann, 1907 (BISTONI \& HuED, 2002) na Argentina, $O$. fugitiva Cope, 1870 (Pеtry et al., 2003) na Amazônia
Central, Odontostilbe sp. (Luz-Agostinho et al., 2006) no reservatório Corumbá, e H. yatai (HIRANo \& AzEvEDo, 2007) em tributários do rio Ibicui. A tendência à herbivoria apresentada por $C$. heterura e $S$. piaba pode ser evidenciada em outros trabalhos que relatam a preferência dos Cheirodontinae por itens de origem vegetal. Entre estes, podemos citar os referentes a $S$. notomelas, classificada como algívora por Luzz et al. (1998) em riachos da bacia do rio Paraná e por CASATTI et al. (2003) no reservatório Rosana. Além disso, Malabarba (2003) atribuiu hábitos herbívoros aos Cheirodontinae baseado no conjunto morfológico dos dentes.

No entanto, diversos autores classificaram espécies de Cheirodontinae em diferentes categorias alimentares, havendo semelhança nos itens ingeridos, porém com diferenças nas frequências de ingestão (DESTEFANIS \& Freyre, 1972; ANGERMEIER \& KarR, 1983; Escalante, 1987; Roche et al., 1993; Alvim et al., 1997, 1998; GASPAR DA Luz et al., 2001; CAZORLA et al., 2003; CASTRO et al., 2004; Melo et al., 2004; Poullly et al., 2006).

Pode-se inferir, então, que o hábito alimentar das espécies desta subfamília mostra uma tendência à onivoria na maioria dos casos, sendo os itens consumidos muito semelhantes, diferindo apenas na proporção e/ou na frequência com que são ingeridos. Além disso, a divergência ao se tentar definir uma classificação alimentar entre os Cheirodontinae pode estar relacionada às diferenças na disponibilidade dos alimentos nos diferentes locais e épocas do ano em que foram estudadas por outros autores.

A sobreposição alimentar entre as espécies foi elevada durante todo o período estudado, possivelmente por se alimentarem basicamente dos mesmos itens. De fato, é de se esperar que espécies com semelhanças morfológicas apresentem dietas também semelhantes (Ross, 1986). Quando se analisa a sobreposição alimentar entre as espécies, a competição interespecífica é tida como um fator importante na partição dos recursos (GERKING, 1994). No entanto, para os Cheirodontinae do rio Ceará Mirim, as relações competitivas, em termos alimentares, aparentemente não são tão importantes e evidentes, o que contradiz as expectativas, quando se leva em consideração o princípio da exclusão competitiva como premissa. Diante desse fato, duas hipóteses poderiam explicar a coexistência dessas espécies no mesmo ambiente. A primeira seria com relação aos recursos alimentares estarem em abundância o suficiente para mantê-las nestas condições, não sendo causa de competição. A segunda hipótese seria que essas espécies poderiam apresentar algumas estratégias de segregação a nível espacial ou temporal para evitar a competição, se alimentando em diferentes estratos na coluna d'água e em horários diferentes (WoOtTon, 1990).

Considerando-se essas hipóteses associadas aos resultados que evidenciam a ausência de competição por alimento entre estas espécies no rio Ceará Mirim, podese inferir a existência de um padrão alimentar entre os peixes desta subfamília, relacionado ao tamanho diminuto das espécies, à morfologia trófica semelhante ou mesmo representar um padrão relacionado à ancestralidade comum das espécies deste grupo. 
Agradecimentos. As autoras agradecem ao Dr. Luiz R. Malabarba pelas contribuições ao desenvolvimento deste trabalho, ao Dr. Hélio C. B. Gurgel do Laboratório de Ecologia e Fisiologia de Peixes (UFRN) por ceder o material coletado e aos colegas do Laboratório de Ictiologia (UFRGS) pelo auxílio e sugestões no decorrer deste estudo.

\section{REFERÊNCIAS BIBLIOGRÁFICAS}

Abrams, P. 1980. Some comments on measuring niche overlap. Ecology 61(1):44-49.

Alvim, M. C. C.; Maia-Barbosa, P. M. \& Alves, C. B. M. 1997. Alimentação de Holoshesthes heterodon (Teleostei: Cheirodontinae) do Reservatório da Usina Hidrelétrica Cajuru - MG, em relação ao nível da água. Acta Limnologica Brasiliensia 9:45-54.

Angermeier, P. L. \& KarR, J. R. 1983. Fish communities along environmental gradients in a system of tropical streams. Environmental Biology of Fishes 9:117-135.

Arcifa, M. S.; Froenlich, O. \& Northcote, T. G. 1988. Distribution and feeding ecology of fishes in a tropical Brazilian reservoir. Memorias de la Sociedad de Ciencias Naturales La Salle 48:301-326.

Bistoni, M. A. \& Hued, A. C. 2002. Patterns of fish species richness in rivers of the central region of Argentina. Brazilian Journal of Biology 62(4b):753-764.

Britski, H. A.; Silimon, K. Z. S. \& Lopes, B. S. 1999. Peixes do Pantanal: manual de identificação. Brasília, Embrapa. $184 \mathrm{p}$.

Casatti, L. 2004. Ichthyofauna of two streams (silted and reference) in the upper Paraná river basin, southeastern Brazil. Brazilian Journal of Biology 64(4):757-765.

Casatti, L.; Mendes, H. F. \& Ferreira, K. M. 2003. Aquatic macrophytes as feeding site for small fishes in the Rosana Reservoir, Paranapanema River, southeastern Brazil. Brazilian Journal of Biology 63(2):213-222.

Castro, R. M. C.; Casatti, L.; Santos, H. F.; Melo, A. L. A.; Martins, L. S. F.; Ferreira, K. M.; Gibran, F. Z.; Benine, R. C.; Carvalho, M.; Ribeiro, A. C.; Abreu, T. X.; Bockmann, F. A.; Pelição, G. Z.; Stopíglia, R. \& Langeani, F. 2004. Estrutura e composição da ictiofauna de riachos da bacia do Rio Grande no Estado de São Paulo, Sudeste do Brasil. Biota Neotropica 4(1):1-39.

Cazorla, A. L.; Durán, W. \& Tejera, L. 2003. Alimentación de la Ictiofauna del Río Sauce Grande, Provincia de Buenos Aires, Argentina. Biología Acuática 20:73-79.

Delariva, R. L.; Agostinho, A. A.; Nakatani, K. \& Baumgartner, G. 1994. Ichthyofauna associated to aquatic macrophytes in the upper Paraná River floodplain. Revista Unimar 16(3):41-60.

Destefanis, S. \& Freyre, L. R. 1972. Relaciones tróficas de los peces de la laguna Chascomús con un intento de referenciación ecológica y tratamiento bioestadístico del espectro trófico. Acta Zoologica Lilloana 29:17-33.

Escalante, A. H. 1987. Dieta comparativa de Cheirodon interruptus interruptus (Osteichthyes, Characidae) en ambientes lénticos y lóticos de la provincia de Buenos Aires. Revista del Museo de La Plata, Serie Zoología, 14(152):35-45.

Esteves, K. E. 2000. Interação peixes-perifíton em uma lagoa marginal do rio Mogi Guaçu (SP): uma abordagem experimental. In: Santos, J. E. \& Pires, J. S. R. eds. Estudos Integrados em Ecossistemas: Estação Ecológica de Jataí. São Carlos, Rima Editora. p.851-867.

Gaspar da Luz, K. D.; Abujanra, F.; Agostinho, A. A. \& Gomes, L. C. 2001. Caracterização trófica da ictiofauna de três lagoas da planície aluvial do alto rio Paraná, Brasil. Acta Scientiarum 23(2): 401-407.

Gause, G. F. 1934. The struggle for existence. New York, Hafner. 163p.

Gerking, S. D. 1994. Feeding Ecology of Fish. San Diego, Academic. 416p.

Graça, W. J. \& Pavanelli, C. S. 2007. Peixes da Planície de Inundação do Alto Rio Paraná e Áreas Adjacentes. Maringá, Eduem. 241p.

Granado-Lorencio, C. \& Garcia-Novo, F. 1986. Feeding habits of the fish community in a eutrophic reservoir in Spain. Ekologia Polska 34(1):95-110.
Gulllen, E. \& Granado, C. 1984. Alimentacion de la ictiofauna del embalse de Torrejon (rio Tajo, Caceres). Limnética 1:304-310.

Hardin, G. 1960. The competitive exclusion principle. Science 131:1292-1297.

Hartz, S. M.; Verani, J. R. \& Barbieri, G. 2000. Partilha de recursos entre as espécies de ciclídeos (Teleostei, Perciformes) em uma lagoa no litoral norte do Rio Grande do Sul, Brasil. Biociências 8(1):33-58.

Herrán, R. A. 1988. Análisis de contenidos estomacales en peces: revisión bibliografica de los objetivos y la metodología. Informes Técnicos del Instituto Español de Oceanografía 63:1-73.

Hirano, R. H. \& Azevedo, M. A. 2007. Hábito alimentar de Heterocheirodon yatai (Teleostei, Characidae, Cheirodontinae) de dois tributários do rio Ibicui, Rio Grande do Sul, Brasil. Biociências 15(2): 207-220.

Hynes, H. B. N. 1950. The food of freshwater sticklebacks (Gasterostomus aculeatus and Pigosteus pungitius), with a review of methods used in studies of the food of fishes. Journal of Animal Ecology 19:36-58.

Jacobo, M. A. C. \& Veron, M. C. B. 1995. Relaciones troficas de la ictiofauna de cuencas autoctonas del Chaco Oriental, Argentina. Revista Brasileira de Biologia 55:419-437.

KREBS, C. J. 1989. Ecological methodology. New York, Harper Collins Publishers. 654p.

Lopretto, E. C. \& Tell, G. 1995. Ecosistemas de aguas continentales: metodologias para su estudio. La Plata, Ediciones Sur. 895p.

Lowe-McConnell, R. H. 1999. Estudos ecológicos de comunidades de peixes tropicais. São Paulo, Edusp. 536p

Luiz, E. A.; Agostinho, A. A.; Gomes, L. C. \& Hahn, N. S. 1998. Ecologia trófica de peixes em dois riachos da bacia do Rio Paraná. Revista Brasileira de Biologia 58:273-285.

Luz-Agostinho, K. D. G.; Bini, L. M.; Fugi, R.; Agostinho, A. A. \& JúlIO JÚNIOR, H. F. 2006. Food spectrum and trophic structure of the ichthyofauna of Corumbá reservoir, Paraná river Basin, Brazil. Neotropical Ichthyology 4(1):61-68.

Malabarba, L. R. 1998. Monophyly of the Cheirodontinae, characters and major clades (Ostariophysi: Characidae). In: Malabarba, L. R.; Reis, R. E.; Vari, R. P.; Lucena, Z. M. S. \& Lucena, C. A. S. eds. Phylogeny and Classification of Neotropical Fishes. Porto Alegre, Edipucrs. p.193-233. . 2003. Subfamily Cheirodontinae. In: Reis, R. E.; Kullander, S. O. \& Ferraris JR., C. J. eds. Check List of the Freshwater Fishes of South and Central America. Porto Alegre, Edipucrs. p.215-221.

Margalef, R. 1986. Ecologia. Barcelona, Omega Ediciones. 951p.

Melo, C. E.; Machado, F. A. \& Pinto-Silva, V. 2004. Feeding habits of fish from a stream in the savanna of Central Brazil, Araguaia Basin. Neotropical Ichthyology 2(1):37-44.

Needham, J. G. \& Needham, P. R. 1978. Guía para el estudio de los seres vivos de las aguas dulces. Barcelona, Editorial Reverte. $131 \mathrm{p}$.

Nelson, J. S. 2006. Fishes of the world. New York, John Wiley. $601 \mathrm{p}$.

Pelicice, F. M. \& Agostinho, A. A. 2006. Feeding ecology of fishes associated with Egeria spp. patches in a tropical reservoir, Brazil. Ecology of Freshwater Fish 15:10-19.

Petry, P.; Bayley, P. B. \& Markle, D. F. 2003. Relationships between fish assemblages, macrophytes and environmental gradients in the Amazon River floodplain. Journal of Fish Biology 63:547-579.

Pianka, E. R. 1994. Evolutionary ecology. New York, Harper Collins College. $416 p$

Poullly, M.; Barrera, S. \& Rosales, C. 2006. Changes of taxonomic and Trophic structure of fish assemblages along an environmental gradient in the Upper Beni watershed (Bolivia). Journal of Fish Biology 68:137-156.

Roche, K. F.; Sampaio, E. V.; Teixeira, D.; Matsumura-Tundisi, T.; Tundisi, J. G. \& Dumont, H. J. 1993. Impact of Holoshesthes heterodon Eigenmann (Pisces: Characidae) on the plankton community of a subtropical reservoir: the importance of predation by Chaoborus larvae. Hydrobiologia 254:7-20.

Ross, S. T. 1986. Resource partitioning in fish assemblages: a review of field studies. Copeia 2:352-388. 
Sabino, J. \& Castro, R. M. C. 1990. Alimentação, período de atividade e distribuição espacial dos peixes de um riacho da Floresta Atlântica (sudeste do Brasil). Revista Brasileira de Biologia 50(1):23-36.

SchöEnER, T. W. 1974. Resource partitioning in ecological communities. Science 185:27-39.

Smith, W. S.; Petrere Jr., M. \& Barrella, W. 2003. The fish fauna in tropical rivers: The case of the Sorocaba river basin, SP, Brazil. Revista de Biologia Tropical 51(3):769-782.
Weatherley, A. H. 1972. Growth and ecology of fish populations. London, Academic. 239p.

Wootton, R. J. 1990. Ecology of teleost fishes. London, Chapman and Hall. 404p.

Zar, J. H. 1999. Biostatistical Analysis. New Jersey, PrenticeHall. 663p.

ZARET, T. M. \& RAND, A. S. 1971. Competition in tropical stream fishes: support for the competitive exclusion principle. Ecology 52(2):336-342. 